\title{
The Application of Modern Algebra and Its Teaching Value are discussed by Vehicle Restrictions
}

\author{
Yan Zhao \\ Xi'an University of Arts and Science, Department of Mathematics, 710065
}

Keywords: Vehicle restriction; Residue class of m; Equivalence class; Congruence

\begin{abstract}
Modern algebra is Abstract algebra, the basis of modern mathematics. Mathematics is an important component of higher education. When speak of math, Many people think it is very far away from our life. But our life is inextricably linked with mathematics actually. From motor vehicle restriction scheme in Xi'an, Shaanxi province, this paper discusses vehicle classification by license plate number, and leads to the concept of the "residual class" in algebra, follow the principle of "no missing, no repeating", and then, We defined operations in the module m equivalence class; examine the nature of the operation, special elements, And we consider their application in real life, and think about other classification methods for vehicle limits, and their advantages and disadvantages. Finally, this paper makes a brief analysis of the teaching value of the Modern algebra.
\end{abstract}

In autumn and winter, the worsening environment and the smog continues to be severe, in order to reduce emission of vehicle pollutants effectively and improve urban air quality continuously, the government of Xi'an has adopted the traffic management measures during the peak period from November 4, 2016.

"Specific requirements as follow: From November 4, 2016, for all the Shaanxi A and the field book brand vehicle, it would implement some traffic control measures according to the license plate number in work day. During the heavy pollution weather emergency response III period, it would limit two 'tail number' daily. Limiting the number of digits is corresponding to the date of the Gregorian calendar. For example, it limited 1 and 6 on 11 date, it limited 2 and 7 on 12 date, it limited 3 and 8 on 13 date, it limited 4 and 9 on 14 date, it limited 5 and 0 on 15 date, it limited 1 and 6 on 16 date, and so on. Statutory holidays and public holidays are unlimited. "Tail number" is the last Arabic numerals on motor vehicle plate. If last one is alphabet, the final Arabic number is executed as the 'tail number'." [1]

In 2017, the motor vehicle restriction policy of Xi 'an had changed, " Limit time and rules: from November 20,2017 to March 15,2018, Each day is limited to motor vehicle of two license plates (including temporary) in 7:00-20:00. ( Statutory holidays and public holidays are unlimited.) If last one is alphabet, the final Arabic number is executed as the 'tail number'. For example, it limited 1 and 6 on Mondays, it limited 2 and 7 on Tuesdays, it limited 3 and 8 on Wednesdays, , it limited 4 and 9 on Thursdays, it limited 5 and 0 on Fridays, and so on." [2]

2016 year ,each number that has been restricted as follow: 4 and 9、5and 0、1 and 6 were limited to two days; 2 and 7、3and 8 were limited to only one day. If it start limiting again on December 5th, it means 5and 0, which the last time restricted twice, would become this round limit line start of car number again. It would be limited the third time. Through the records and find this post, in the same time period, the two groups number were limited once, the other group number were limited three times. Private cars are restricted to different times due to the same smog! It was unfair for such limit plan! The restriction scheme had become a way to limit the practice of the week on 2017. It is consistent with the people's working habits, and it is also convenient to remember, and the number of limits per vehicle is also basically the same. In the meantime, the number of cities restricted to motor vehicles is increasing because of environmental pollution such as Beijing、Shenzhen、Wuhan、Chengdu、Shijiazhuang...... The traffic restrictions will become the new normal of China's large and medium-sized cities. But, how to explain the traffic control in mathematical? We will discuss it algebraically. 
We will be classified the number which need limited motor vehicles followed the principle of fairness. Limiting the number of days has the same extent for each restricted vehicle. In a limited period, the vehicle cannot repeat the limit line, nor can it be missed, the best way is the same number of days. This is the principle that the set classification in our algebra----"no leaking, no missing", and there is the same number of elements in the subset.

Definition. $1^{[3]}$ Set A, divided A into number of non-empty subsets, so that each element in the subset belongs to and belongs to only A subset, and all of these subsets are called classification of A. Each subset is called A class. In other words, if the non-empty subset of A $S=\left\{A_{i} \mid i \in I\right\}$ is A classification, if and only if $\mathrm{S}$ satisfied the following properties:

(1) $\bigcup_{i \in I} A_{i}=A$

(2) when $i \neq j, A_{i} \cap A_{j}=\varnothing$,

That is they are not classification while sets is disjoint each other.

From this, we are considering putting the number which is of motor vehicle license plates that need to be limited in $\mathrm{Xi}$ 'an into a collection. The number of elements in a collection is the number of vehicles that need to be limited. In this set, we classify it, and the principle is no leaking, no missing. Try to make the same number of elements in each subset. Therefore, we discuss the classification of equivalence relation.

Proposition. $1^{[3]}$ Given a set A, $A=Z, m \in N$, make $R_{m}=\{(a, b)|a, b \in Z, m| a-b\}$,so $R_{m}$ is a equivalence relation in set of integer $Z$. A classification of $Z$ can be determined by this equivalence relation. So a is equal to $\mathrm{b}$ with respect to $\bmod \mathrm{m}$.

Proof. Obviously, $R_{m}$ is a subset of $Z \times Z, R_{m}$ is a equivalence relation in set of integer $Z$.

Because (1) $\forall a \in Z, m \mid a-a$,so $a R_{m} a$,

it satisfied relation of self-contrary;

(2) $\forall a, b \in Z$, if $a R_{m} b$, then $m \mid a-b$, and $m \mid b-a$,so $b R_{m} a$,

it satisfied relation of symmetrical;

(3) $\forall a, b, c \in Z$, if $a R_{m} b, b R_{m} c$, then $m|a-b, m| b-c$,so $m \mid(a-b)+(b-c)$,

it is $m \mid a-c$,So $a R_{m} c$,

It satisfied relation of transferable.

Therefore, ${ }^{R_{m}}$ is a equivalence relation in set of integer $Z$,

The equivalent class determined is.

$[0]=\{\cdots-2 m,-m, 0, m, 2 m \cdots\}$,

$[1]=\{\cdots-2 m+1,-m+1,1, m+1,2 m+1 \cdots\}$,

$[2]=\{\cdots-2 m+2,-m+2,2, m+2,2 m+2 \cdots\}$

$[m-1]=\{\cdots-m-1,-1, m-1,2 m-1,3 m-1 \cdots\}$.

$R_{m}$ is called the congruence of the $\bmod m$, The equivalent class determined by $R_{m}$ is called the module $m$ residue class. The quotient set about $Z_{\text {and }} R_{m}$ is: $Z_{m}=\{[0],[1],[2], \cdots[m-1]\}$, are some collections of $m$ different residual classes.

It's easy to see, the rules of Xi'an vehicle restriction plan in 2016 years is that the last digit of the vehicle number and the date of the Gregorian calendar mod 5 are limited (not limited on the weekend).It is residual class of module 5 in algebra_ $Z_{5}$. The rules of Xi' an vehicle restriction plan in 2017 years is that the last digit of the vehicle license plate and the days of week mod 5 are 
limited (not limited on the weekend). It is also residual class of module 5 in algebra $-Z_{5}$.

And then, we will discuss the groups of remaining model $\mathrm{m}$.

Proposition 2 In set $Z_{m}=\{(a, b)|a, b \in Z, m| a-b\}$, Provisions of $[a]+[b]=[a+b],[a] \cdot[b]=[a b]$, Among them $[a],[b] \in Z_{m}$,so $\left(Z_{m},+\right),\left(Z_{m}, \bullet\right)$ are all exchange of Semi group.

Because Quotient set $Z_{m}=\{[0],[1],[2], \cdots[m-1]\}$ has a zero element ${ }^{[0]}$, has a negative element $[-a]$. About add operation $\left(Z_{m},+\right)$ would be a Add group. It called the residual class of $\mathrm{m}$.

With the concept and related properties of the residual classes of $\mathrm{m}$ in algebra, it is more convenient for us to study the restriction of vehicles. At present, urban vehicle restriction form in addition to what we mentioned above----- the vehicle type of bottom Numbers and calendar dates and 5 congruence, is die five remaining on the algebra class---- In addition, Beijing and other cities are restricted to the odd-even number (weekend limit), which is the residue class of model 2 and the most limited number of vehicles.

For example, the residual class of module 3, It can be specified that the last digit of the license plate is limited to the same day as $3^{[6]}$. In particular, the last digit of the license plate is divided by 3 remainder 1, limiting on Monday and Thursday. The remaining 2 is restricted on Tuesday and Friday; The remaining 3 is restricted on Wednesday and Saturday; There is no limiting on the Sunday. That is the vehicles were restricted on Monday and Thursday that tail Numbers are 1, 4, 7; the vehicles were restricted on Friday and Tuesday that tail Numbers are 2, 5, 8; the vehicles were restricted on Wednesday and Saturday that tail Numbers are 0, 3, 6, 9. Compared to the previous two restrictions, this plan is designed to be limited due to the equivalent class. But the number of elements in each equivalence class is different, It lead to limit the vehicle every day 3, 4 Numbers, it is unfair enough. It has some flaws what is limited the number $0,3,6,9$ on Wednesday and Saturday, especially on Saturday, a weekend, it is not necessary for restricted the maximum number of cars. It would affect the people's life, a bit not practical. For example ${ }^{[7]}$, considering the remaining class of module 10 , the rule is: the vehicle is restricted if the tail number of the license plate is the same as the calendar date. Specifically, the vehicle would be restricted on Number 1, 11, 21, 31 a month if it license plate number is 1 ; the vehicle would be restricted on Number 2, 12, 22 a month if it license plate number is 2; and so on. The advantage of this limited scheme is that it is easy to remember and limit the less vehicles per day. Faults is also evident, there are 31 days on some month, vehicles which license plate number is 1 will be limit more than the others one day per month, vehicles which license plate number is 0 or 9 will be limit less than the others one day on February. It can't follow the principle of fairness. How to solve this problem? We can do no limiting on the 31 st of the month, but it can lead to traffic chaos, air pollution and other social problems.

All in all, there are many methods of traffic restrictions other than the ones we listed above. We will find the most rational and effective way for vehicles in every city and in different period of time based on the principle of fairness if the scale of city, transportation condition and environmental pollution are taken into consideration.

Modern algebra, a systematic mathematic subject in various abstract Axiomatic algebra, is characterized by high degree of abstraction, intensity of reasoning and wide range of teaching, so it is tedious and hard to grasp for most students. Currently, the subject is instructed in a way of explaining concepts, theories and examples focusing on the fields of set of numbers 、 matrix, which is in no effective relation to the real world and is not applicable. Now, by combining the realistic problems with the abstract Modern algebra concepts, we aim at training students to solve these problems by applying the basic Modern algebra theories, provide more ways of thinking, motivate their interests and enhance their comprehensive capacity by guiding them to compare and associate the relationships between subjects, and explore the ways to solve the problem and comprehensively improve students', synthetic ability and quality. 


\section{Project Information}

2014 Shaanxi education department research project.

Name: some famous and mean studies in number theory; Item no. 14JK2134.

\section{References}

[1] from the first edition of Huashang Daily, November 4, 2016.

[2] xi 'an bus information December 12, 2017.

[3] Zhu ping, Li Bo, Zhou Yuan, modern algebra [M], Beijing: science press, 2001.8.

[4] Zhang He-rui basic algebra foundation [M] Beijing: higher education press, 2000.5.

[5] Wang Yuan discusses the formulation of public policy from Beijing vehicle restriction policy [J] Chinese and foreign entrepreneurs 2011 (08).

[6] Zhao Yan Vehicle restriction And residue class of $\mathrm{m}$ [J] Technology outlook 2017 (31).

[7] Wang Ya-nan, Wang yu-qiong the impact of the restrictions on residents' travel and their effectiveness analysis [J] urban public transport 2011.

[8] Yu Jia-fei sees effects and risks from the practice of motor vehicle restrictions $[\mathrm{J}]$ transportation and transportation 2011.

[9] Wu Qiang-Zhao, Zhao Hong-wei single - and double - number limited test IT management [J] computer world. 